\title{
Prevalence of Prostatitis-Like Symptoms and Outcomes of NIH-CPSI in Outpatients with Lifelong and Acquired PE: Based on a Large Cross-Sectional Study in China
}

\author{
Daofang Zhu, Xianming Dou, Liang Tang, Dongdong Tang, Guiyi Liao, \\ Weihua Fang, and Xiansheng Zhang \\ Department of Urology, The First Affiliated Hospital of Anhui Medical University, Hefei City, Anhui Province, China \\ Correspondence should be addressed to Xiansheng Zhang; xianshengzhangzxs@yeah.net
}

Received 15 March 2017; Accepted 19 June 2017; Published 7 August 2017

Academic Editor: Kurt G. Naber

Copyright ( 92017 Daofang Zhu et al. This is an open access article distributed under the Creative Commons Attribution License, which permits unrestricted use, distribution, and reproduction in any medium, provided the original work is properly cited.

Premature ejaculation (PE) is one of the most common sexual dysfunctions, which were associated with prostatitis-like symptoms (PLS). We intended to explore the prevalence of prostatitis-like symptoms and outcomes of National Institutes of Health-Chronic Prostatitis Symptom Index (NIH-CPSI) scores in outpatients with lifelong (LPE) and acquired premature ejaculation (APE). From December 2013 to December 2015, a total of 498 consecutive heterosexual men with PE and 322 male healthy subjects without PE were enrolled. Each of them completed a detailed questionnaire on demographics information, sexual and medical histories, and the NIH-CPSI. Assessment of NIH-CPSI and definition of PLS and PE were used to measure the PLS and NIH-CPSI scores and ejaculatory function for all subjects. Finally, a total of 820 subjects (including 498 men in PE group and 322 men in control group) were enrolled in our study. The mean ages were significantly different between PE and no PE groups. Men with PE reported worse PLS and higher NIH-CPSI scores $(P<0.001$ for all). Similar findings were also observed between men with LPE and APE. Men with APE also reported higher rates of PLS and scores of NIH-CPSI $(P<0.001$ for all). Multivariate analysis showed that PLS and NIH-CPSI scores were significantly associated with PE.

\section{Introduction}

Premature ejaculation (PE) is one of the most common sexual dysfunctions, which affects about $20 \% \sim 30 \%$ of male population due to its different definitions [1-4]. Recently, the International Society for Sexual Medicine (ISSM) provided a new definition of PE (lifelong PE [LPE] and acquired PE [APE]) [5]. They included three main characteristics: (1) intravaginal ejaculatory latency time (IELT) always or nearly always about or less than a minute from the first sexual experience (LPE) or a clinically significant and bothersome reduction of IELT, often about or less than 3 minutes (APE); (2) lack of ability to delay ejaculation on all or nearly all vaginal penetrations; and (3) negative personal consequences, such as distress, bothering, frustration, and/or the avoidance of sexual intimacy.

In addition, previous studies have shown that chronic prostatitis (CP) might be contributed to PE. Screponi et al. found that the percentage of prostatic inflammation and chronic bacterial prostatitis in patients with PE was 56.5\% and $47.8 \%$, respectively, and there were significant differences between PE patients and controls [6]. The other large observational survey in China showed that PE (especially APE patients) reported worse National Institutes of HealthChronic Prostatitis Symptom Index (NIH-CPSI) scores and lower IELT than men without PE complaints [1]. Similarly, Liang et al. found that the PE group reported worse NIHCPSI scores, and the percentage of PE was $64.1 \%$ and $36.9 \%$ in the prostatitis-like symptom (PLS) and CP group, respectively [7].

Although the associations between $\mathrm{CP}$ and PE have been widely studied, they were still not clear. In addition, based on the new definition of LPE and APE, studies on the issues in China were rare. Hence, our study intended to explore the prevalence of PLS and outcomes of NIH-CPSI scores in outpatients with LPE and APE (Table 2). 


\section{Subjects and Methods}

An observational and cross-sectional field survey was conducted in the urological department of the Affiliated Hospital of Anhui Medical University. From December 2013 to December 2015, a total of 498 consecutive heterosexual men with PE (including 142 lifelong PE and 356 acquired PE) were recruited from the andrology outpatient's clinic. In addition, 322 male healthy volunteers without PE were enrolled from the health examination center.

To be included in the study, all subjects had to meet the following criteria: (1) age $\geq 18$ years, (2) being in a heterosexual, stable relationship for at least 6 months, and (3) being able to read and speak Chinese. Their sexual and medical history was carefully evaluated by an experienced clinician. Men on medication that could have affected their ejaculation were excluded.

Patients were diagnosed as having LPE and APE according to evidence-based definitions recommended by ISSM. Since several subjective and sensitive personal questions were included in this study, a presurvey was given to a small sample $(N=30)$ to modify the questionnaire and make it comprehensive and easily understood. Before the survey, all subjects were informed about the study and those who participated were asked to provide written consent. Then they completed a questionnaire, including demographic information (e.g., age, education, and occupation), sexual and medical histories (e.g., self-estimated IELT), and the Chinese version of NIH-CPSI [8]. This survey was reviewed and approved by the Anhui Medical University Research Subject Review Board.

Assessment of NIH-CPSI is a reliable, convenient, selfadministered index that is widely used across scientific research and clinical studies (including pain symptoms [total of items 1-4], urinary symptoms [total of items 5 and 6], and Quality of life [QOL] impact [total of items 7-9]) [9]. The Chinese version of NIH-CPSI was widely used in the previous studies in China [7, 8, 10-12]. Based on the total of items 1-9, the severity of CPPS was classified as mild (10-14 points), moderate (15-29 points), or severe ( $\geq 31$ points). In addition, according to Nickel et al. [9], perineal and/or ejaculatory pain/discomfort and a total pain score of NIH-CPSI $\geq 4$ were used to identify men with PLS. Mild symptoms were defined by pain scores of 4 to 7 and moderate or severe symptoms by scores of 8 or greater.

All data were analyzed using the SPSS software version 13.0 (SPSS Inc., Chicago, IL, USA). Descriptive statistics were used to summarize the subjects' characteristics. Data were expressed as mean \pm standard deviation or number (percentage) when appropriate. Chi-squared test was used to compare categorical data. The independent $t$-test was used to compare numerical data. The analysis of covariance was used to assess the factors associated with PLS. In addition, the multivariate analysis (adjusted by age, BMI scores, smoking, and exercise) was performed to assess the association between PLS, NIH-CPSI scores, and PE. Odds Ratio (OR) and Confidence Interval (CI) were used to evaluate their associated strength. For all tests, $P<0.05$ was considered statistically significant.

\section{Results}

A total of 820 subjects (including 498 men in PE group and 322 men in control group) were enrolled in our study. The mean ages in PE (LPE, 28.51\%; APE, 71.49\%) and no $\mathrm{PE}$ groups were $43.41 \pm 9.45$ and $32.72 \pm 8.86$ years. In addition, the mean self-estimated IELT in PE groups was $1.49 \pm 0.56$ minutes, whereas that in control group was $3.16 \pm 1.49$ minutes. Moreover, the mean self-estimated IELT in men with LPE and APE were $0.84 \pm 0.25$ minutes and $1.75 \pm 0.76$ minutes, respectively. Differences between PE and no PE groups were observed with regard to age, body mass index (BMI), self-estimated IELT, and rates of smoking and exercise $(P<0.001$ for all). Similarly, the differences were also presented between LPE and APE groups. Detailed demographic characteristics of men with and without PE were summarized in Table 1.

Also, significant difference was found between PE and no PE groups, when we analyzed the severity of PLS and prostatitis symptoms and NIH-CPSI scores $(P<0.001$ for all). The incidence of PLS in men with PE was $36.54 \%$, whereas that in no PE group was $13.36 \%$. The mean NIH-CPSI scores in LPE and APE were $24.47 \pm 8.44$ and $33.26 \pm 9.21$, respectively. That is to say, men with $\mathrm{PE}$ reported worse PLS and higher NIH-CPSI scores. In addition, similar findings were also observed between men with LPE and APE. Men with APE also reported higher rates of PLS and scores of NIHCPSI (including total and subdomain scores) $(P<0.001$ for all).

Finally, with the analysis of covariance, significant associations were found between PLS and some factors (including age, BMI scores, smoking, exercise, and NIH-CPSI scores) $(P<0.001$ for all $)$. However, the multiple logistic regression (adjusted by age, BMI scores, smoking, and exercise) showed the association between PLS and NIH-CPSI scores and PE (PLS $[\mathrm{OR}=2.75, \mathrm{CI}=2.26-4.33]$ and NIH-CPSI scores $[15-30:$ OR $=1.72, \mathrm{CI}=1.43-2.65 ; 31-43$ : $\mathrm{OR}=2.83, \mathrm{CI}=$ 2.45-4.52]) (Table 3).

\section{Discussion}

Based on the new definition of PE, this is the first study to investigate the incidence of PLS and outcomes of NIH-CPSI in outpatients with LPE and APE. In addition, the new PE classification provides a better perspective of the epidemiology, pathophysiology, etiology, and treatment of various forms of PE. In our study, we found that the prevalence of PLS and NIH-CPSI scores in PE group were higher than those in no PE group. In addition, patients with APE reported higher incidence of PLS and NIH-CPSI scores than men with LPE.

PE was known to be a multifactorial sexual dysfunction [10, 13-15]. Previous studies have showed that it might be associated with prostatitis symptoms. After evaluating a consecutive series of 244 men with couple infertility, Lotti et al. [16] found that PE (evaluated by PE diagnostic tool [PEDT]) was positively associated with prostatitis symptoms. PEDT score was related to the total and subdomain scores of NIH-CPSI. In an observational study of 1,5000 Chinese men, Liang et al. [7] found that $64.07 \%$ of patients with 
TABLE 1: Demographic characteristics in men with and without PE.

\begin{tabular}{|c|c|c|c|c|c|c|c|}
\hline Factors & $\operatorname{ALL}(n=820)$ & PE group $(n=498)$ & No PE group $(n=322)$ & $P^{*}$ value & $\operatorname{LPE}(n=142)$ & $\operatorname{APE}(n=356)$ & $P^{* *}$ value \\
\hline Age, years & $39.21 \pm 9.25$ & $43.41 \pm 9.45$ & $32.72 \pm 8.86$ & $<0.001$ & $38.72 \pm 9.17$ & $45.28 \pm 10.83$ & $<0.001$ \\
\hline BMI, scores & $25.06 \pm 3.14$ & $26.59 \pm 3.47$ & $22.69 \pm 2.75$ & $<0.001$ & $23.74 \pm 3.82$ & $27.73 \pm 3.65$ & $<0.001$ \\
\hline IELT, minutes & $2.15 \pm 1.28$ & $1.49 \pm 0.56$ & $3.16 \pm 1.49$ & $<0.001$ & $0.84 \pm 0.25$ & $1.75 \pm 0.76$ & $<0.001$ \\
\hline Smoking & $487(59.39 \%)$ & $336(64.47 \%)$ & $151(46.89 \%)$ & $<0.001$ & $76(53.52 \%)$ & $260(73.03 \%)$ & $<0.001$ \\
\hline Exercise & $347(42.32 \%)$ & $184(36.95 \%)$ & $163(50.62 \%)$ & $<0.001$ & $66(46.48 \%)$ & $118(33.15 \%)$ & $<0.001$ \\
\hline Educational status & & & & 0.612 & & & 0.994 \\
\hline Others & $117(14.27 \%)$ & $67(13.45 \%)$ & $50(15.53 \%)$ & & $20(14.08 \%)$ & $47(13.20 \%)$ & \\
\hline Primary education & $171(20.85 \%)$ & $100(20.08 \%)$ & $71(22.05 \%)$ & & $28(19.72 \%)$ & $72(20.22 \%)$ & \\
\hline High school & $330(40.24 \%)$ & $202(40.56 \%)$ & $128(39.75 \%)$ & & $57(40.14 \%)$ & $145(40.73 \%)$ & \\
\hline Higher education & $202(24.63 \%)$ & $129(25.90 \%)$ & $73(22.67 \%)$ & & $37(26.06 \%)$ & $92(25.84 \%)$ & \\
\hline Occupational status & & & & 0.463 & & & 0.880 \\
\hline Student & $192(23.41 \%)$ & $110(22.09 \%)$ & $82(25.47 \%)$ & & $32(22.54 \%)$ & $78(21.91 \%)$ & \\
\hline Unemployed & $198(24.15 \%)$ & $121(24.30 \%)$ & 77 (23.91\%) & & $31(21.83 \%)$ & $90(25.28 \%)$ & \\
\hline Employed & 333 (40.61\%) & $202(40.56 \%)$ & $131(40.68 \%)$ & & $60(42.25 \%)$ & $142(39.89 \%)$ & \\
\hline Retired & 97 (11.83\%) & 65 (13.05\%) & $32(9.94 \%)$ & & $19(13.38 \%)$ & $46(12.92 \%)$ & \\
\hline
\end{tabular}

$\mathrm{PE}=$ premature ejaculation; $\mathrm{BMI}=$ body mass index; $\mathrm{IELT}=$ intravaginal ejaculatory latency time; $\mathrm{LPE}=$ lifelong $\mathrm{PE} ; \mathrm{APE}=$ acquired $\mathrm{PE}^{*} \mathrm{D}$ Differences between PE complaint and no PE complaint were assessed by Chi-square test or $t$-test, as appropriate. ${ }^{* *}$ Differences between LPE and APE were assessed by Chi-square test or $t$-test, as appropriate.

TABLE 2: Outcomes of PLS and NIH-CPSI in men with LPE/APE.

\begin{tabular}{|c|c|c|c|c|c|c|c|}
\hline & $\operatorname{ALL}(n=820)$ & PE group $(n=498)$ & No PE group $(n=322)$ & $P^{*}$ value & $\operatorname{LPE}(n=142)$ & $\operatorname{APE}(n=356)$ & $P^{* *}$ value \\
\hline PLS & & & & $<0.001$ & & & $<0.001$ \\
\hline Mild (4-7) & $134(16.34 \%)$ & $102(20.48 \%)$ & $32(9.94 \%)$ & & $23(16.20 \%)$ & $79(22.19 \%)$ & \\
\hline Moderate or severe $(\geq 8)$ & $91(11.10 \%)$ & $80(16.06 \%)$ & $11(3.42 \%)$ & & $18(12.68 \%)$ & $62(17.42 \%)$ & \\
\hline \multicolumn{8}{|l|}{ NIH-CPSI, scores } \\
\hline Total scores & $19.74 \pm 6.85$ & $30.75 \pm 8.89$ & $2.72 \pm 1.08$ & $<0.001$ & $24.47 \pm 8.44$ & $33.26 \pm 9.21$ & $<0.001$ \\
\hline Pain symptoms & $10.44 \pm 4.32$ & $16.44 \pm 5.64$ & $1.15 \pm 0.41$ & $<0.001$ & $14.46 \pm 5.04$ & $17.23 \pm 6.12$ & $<0.001$ \\
\hline Urinary symptoms & $4.92 \pm 1.88$ & $7.43 \pm 3.06$ & $1.03 \pm 0.40$ & $<0.001$ & $5.27 \pm 2.85$ & $8.29 \pm 3.33$ & $<0.001$ \\
\hline Quality of life impact & $4.37 \pm 1.74$ & $6.85 \pm 2.16$ & $0.54 \pm 0.16$ & $<0.001$ & $4.74 \pm 1.45$ & $7.74 \pm 2.36$ & $<0.001$ \\
\hline NIH-CPSI, scores & & & & $<0.001$ & & & $<0.001$ \\
\hline Mild (0-14) & $622(75.85 \%)$ & $314(63.05 \%)$ & $308(95.65 \%)$ & & $98(69.01 \%)$ & $216(60.67 \%)$ & \\
\hline Moderate (15-30) & $142(17.32 \%)$ & $128(25.70 \%)$ & $14(4.35 \%)$ & & $32(22.54 \%)$ & $96(26.97 \%)$ & \\
\hline Severe (31-43) & $56(6.83 \%)$ & $56(11.24 \%)$ & $0(0.00 \%)$ & & $12(8.45 \%)$ & $44(12.36 \%)$ & \\
\hline
\end{tabular}

$\mathrm{PE}=$ premature ejaculation; $\mathrm{LPE}=$ lifelong PE; APE = acquired PE; PLS = prostatitis-like symptoms; NIH-CPSI = National Institute of Health-Chronic Prostatitis Symptoms Index. ${ }^{*}$ Differences between PE and no PE groups were assessed by $t$-test. ${ }^{* *}$ Differences between LPE and APE were assessed by $t$-test.

TABle 3: Association between PLS, NIH-CPSI scores, and PE.

\begin{tabular}{|c|c|c|c|c|}
\hline \multirow{2}{*}{ Factors } & \multirow{2}{*}{$P$ value } & \multirow{2}{*}{$\mathrm{OR}^{*}$} & \multicolumn{2}{|c|}{$95 \%$ CI } \\
\hline & & & Lower & Upper \\
\hline \multicolumn{5}{|l|}{ PLS } \\
\hline No & 1 & & & \\
\hline Yes & $<0.001$ & 2.75 & 2.26 & 4.33 \\
\hline \multicolumn{5}{|c|}{ NIH-CPSI, scores } \\
\hline $0-14$ & $<0.001$ & 1 & & \\
\hline $15-30$ & $<0.001$ & 1.72 & 1.43 & 2.65 \\
\hline $31-43$ & $<0.001$ & 2.83 & 2.45 & 4.52 \\
\hline
\end{tabular}

$\mathrm{PE}=$ premature ejaculation; PLS = prostatitis-like symptoms; NIH-CPSI = National Institute of Health-Chronic Prostatitis Symptoms Index; OR = Odds Ratio; $\mathrm{CI}=$ Confidence Interval. ${ }^{*}$ Date were assessed by the multiple logistic regression, when adjusted by age, BMI scores, smoking, and exercise. 
prostatitis-like symptoms reported $\mathrm{PE}$, significantly more than one might expect in a population ranging from 51 to 60 years. In addition, participants with $\mathrm{PE}$ versus men without PE reported worse NIH-CPSI total (37.2 \pm 4.6 versus $18.2 \pm 5.6$ ) and subdomain scores (including pain symptoms, urinary symptoms, and QOL subscores). Relationships between PE and CP symptoms were obvious in their study. Another study conducted by Bartoletti et al. [17] showed that chronic pelvic pain syndrome (CPPS) had a negative influence on male sexual function. Compared with the control group, the incidence of PE was higher in patients with CPPS. Similar findings were also observed in our survey. Results from our survey showed that men with PE reported higher incidence of PLS than men without PE. In addition, NIH-CPSI scores in $\mathrm{PE}$ groups were higher than those in control group. However, the difference in prevalence rates might be explained by the cultural and religious differences between the Chinese and Western patient populations used in the respective studies.

In addition, association between PLS and age, BMI scores, lifestyle, and NIH-CPSI scores was showed in our study. Men with PLS might report older age, higher BMI scores and $\mathrm{NIH}-\mathrm{CPSI}$ scores, and unhealthy lifestyle (e.g., smoking, no exercise). Because the definition of PLS includes perineal or ejaculatory discomfort and a total pain score of NIHCPSI $\geq 4$, we speculated that factors associated with perineal or ejaculatory discomfort (e.g., age, smoking) and NIHCPSI scores might influence the incidence of PLS. However, previous studies on the issue were few in China, and further studies were needed.

We also investigated the incidence of PLS and outcomes of NIH-CPSI in outpatients with LPE and APE, respectively. Our study showed that APE patients reported higher PLS and NIH-CPSI scores than LPE patients. Similarly, Serefoglu et al. [18] found that the incidence of CP in men with APE were higher than men with LPE. In another study based on 690 middle-aged men complaining of ejaculating prematurely, results reported that the correlation of IPSS with self-estimated IELT of APE patients was stronger than that with LPE patients [19]. Gao et al. [20] also found that negative relationships between total and subdomain scores of NIHCPSI and IELT were stronger in men with APE than LPE. Hence, our results confirmed the above findings, and further studies were also needed.

There are also several limitations in the present study. Subjects participating in this study completed the questionnaires face to face with investigators, and so many patients may exaggerate or reduce their symptoms due to embarrassment when dealing with this sensitive personal problem. Particularly, the NIH-CPSI may influence the accuracy of our findings. Other methods of obtaining data from these patients, such as Internet-based surveys, should be considered for future studies. And we did not take any laboratory examinations and ultrasonography to exclude other diseases, for example, benign prostatic hyperplasia. A further and more accurate definition of $\mathrm{CP}$ is needed to indicate the exact prevalence. Finally, we have chosen the control group from our medical examination center; a community-based investigation should be made in further study for more accurate study.

\section{Conclusion}

Patients with PE had higher incidence of PLS and CP and had higher NIH-CPSI scores than that of the control subjects. Incidence of PLS in the APE group was higher than that of LPE group.

\section{Conflicts of Interest}

All authors have no conflicts of interest.

\section{Authors' Contributions}

Daofang Zhu and Xianming Dou contributed equally to this work.

\section{Acknowledgments}

The authors thank all individuals who provided the information for this study. This study was funded by the National Natural Science Foundation of China (no. 81571429).

\section{References}

[1] J. Gao, X. Zhang, P. Su et al., "Prevalence and impact of premature ejaculation in outpatients complaining of ejaculating prematurely: using the instruments of intravaginal ejaculatory latency time and patient-reported outcome measures," International Journal of Impotence Research, vol. 26, no. 3, pp. 94-99, 2014.

[2] M. Karabakan, A. Bozkurt, E. Hirik et al., "The prevalence of premature ejaculation in young Turkish men," Andrologia, vol. 48, no. 9, pp. 895-899, 2016.

[3] S. W. Lee, J. H. Lee, H. H. Sung et al., "The prevalence of premature ejaculation and its clinical characteristics in Korean men according to different definitions," International Journal of Impotence Research, vol. 25, no. 4, article 160, 2013.

[4] E. C. Serefoglu and T. R. Saitz, "New insights on premature ejaculation: A review of definition, classification, prevalence and treatment," Asian Journal of Andrology, vol. 14, no. 6, pp. 822-829, 2012.

[5] E. C. Serefoglu, C. G. McMahon, M. D. Waldinger et al., "An evidence-based unified definition of lifelong and acquired premature ejaculation: report of the second international society for sexual medicine ad hoc committee for the definition of premature ejaculation," The Journal of Sexual Medicine, vol. 11, no. 6, pp. 1423-1441, 2014.

[6] E. Screponi, E. Carosa, S. M. Di Stasi, M. Pepe, G. Carruba, and E. A. Jannini, "Prevalence of chronic prostatitis in men with premature ejaculation," Urology, vol. 58, no. 2, pp. 198-202, 2001.

[7] C.-Z. Liang, Z.-Y. Hao, H.-J. Li et al., "Prevalence of premature ejaculation and its correlation with chronic prostatitis in Chinese men," Urology, vol. 76, no. 4, pp. 962-966, 2010.

[8] J. Guo, Y.-Q. Zhang, J.-M. Jia et al., "The observation of chronic prostatis patients using the National Institutes of Health Chronic Prostatitis Symptom Index," Zhonghua nan ke xue = National journal of andrology, vol. 8, no. 2, pp. 127-129, 2002.

[9] J. C. Nickel, J. Downey, D. Hunter, and J. Clark, "Prevalence of prostatitis-like symptoms in a population based study using the National Institutes of Health chronic prostatitis symptom index," The Journal of Urology, vol. 165, no. 3, pp. 842-845, 2001. 
[10] J. Gao, X. Zhang, P. Su et al., "Prevalence and factors associated with the complaint of premature ejaculation and the four premature ejaculation syndromes: a large observational study in China," The Journal of Sexual Medicine, vol. 10, no. 7, pp. 18741881, 2013.

[11] D. Tang, X. Zhang, Z. Hao, J. Zhou, and C. Liang, "Prevalence of prostatitis-like symptoms in outpatients with four premature ejaculation syndromes: a study in 438 men complaining of ejaculating prematurely," International Journal of Clinical and Experimental Medicine, vol. 7, no. 7, pp. 1829-1836, 2014.

[12] Y. Wang, L. He, Z. Zhou et al., "The association between metabolic syndrome and the National Institutes of health chronic prostatitis symptom index: results from 1673 men in China," Urology, vol. 82, no. 5, pp. 1103-1107, 2013.

[13] S. E. Althof, "Prevalence, characteristics and implications of premature ejaculation/rapid ejaculation," Journal of Urology, vol. 175, no. 3, pp. 842-848, 2006.

[14] X. Zhang, J. Gao, J. Liu et al., "Prevalence rate and risk factors of depression in outpatients with premature ejaculation," BioMed Research International, vol. 2013, no. 1, Article ID 317468, 2013.

[15] M. Mas, "An update on ejaculation physiology and premature ejaculation definition, prevalence data, and etiology," Semergen/Sociedad Espanola de Medicina Rural y Generalista, vol. 40, no. 1, Supplementary 3, pp. 3-10, 2014.

[16] F. Lotti, G. Corona, G. Rastrelli, G. Forti, E. A. Jannini, and M. Maggi, "Clinical correlates of erectile dysfunction and premature ejaculation in men with couple infertility," The Journal of Sexual Medicine, vol. 9, no. 10, pp. 2698-2707, 2012.

[17] R. Bartoletti, T. Cai, N. Mondaini et al., "Prevalence, incidence estimation, risk factors and characterization of chronic prostatitis/chronic pelvic pain syndrome in urological hospital outpatients in Italy: results of a multicenter case-control observational study," The Journal of Urology, vol. 178, no. 6, pp. 24112415, 2007.

[18] E. C. Serefoglu, O. Yaman, S. Cayan et al., "Prevalence of the complaint of ejaculating prematurely and the four premature ejaculation syndromes: results from the turkish society of andrology sexual health survey," The Journal of Sexual Medicine, vol. 8, no. 2, pp. 540-548, 2011.

[19] X. Zhang, D. Tang, C. Xu et al., "The relationship between selfestimated intravaginal ejaculatory latency time and international prostate symptom score in middle-aged men complaining of ejaculating prematurely in China," The Journal of Sexual Medicine, vol. 12, no. 3, pp. 705-712, 2015.

[20] J. Gao, P. Gao, and Z. Hao, "Comparison of national institutes of health-chronic prostatitis symptom index with international index of erectile function 5 in men with chronic prostatitis/chronic pelvic pain syndrome: a large cross-sectional study in China," BioMed Research International, vol. 2015, 1, Article ID 560239, 2015. 


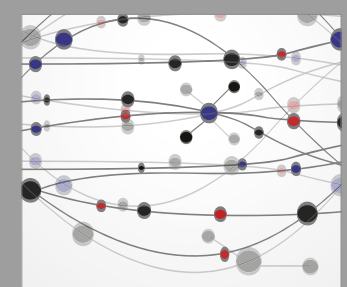

The Scientific World Journal
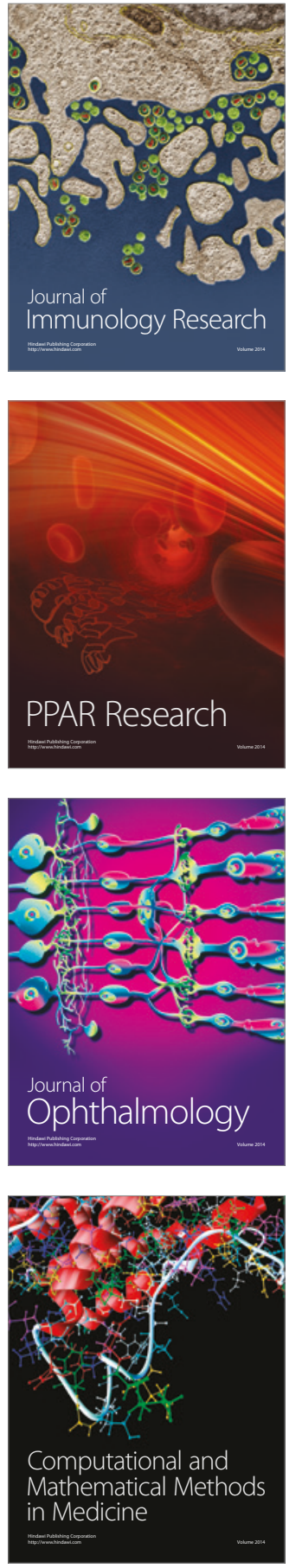

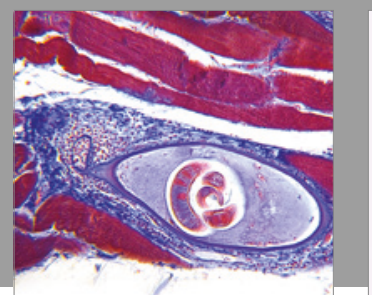

Gastroenterology Research and Practice
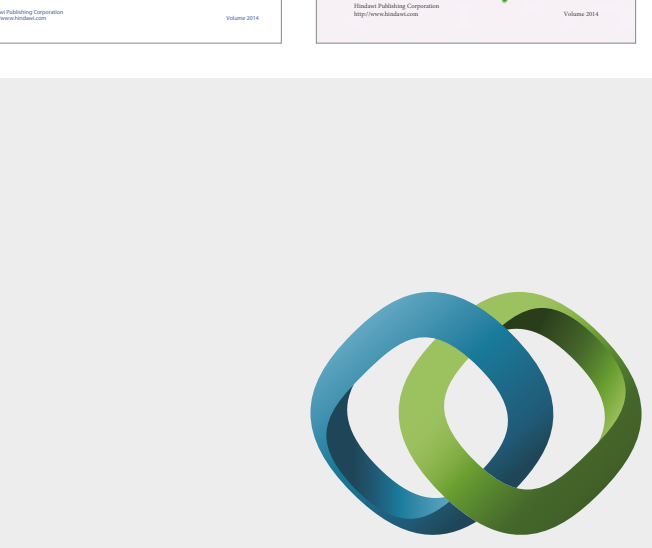

\section{Hindawi}

Submit your manuscripts at

https://www.hindawi.com
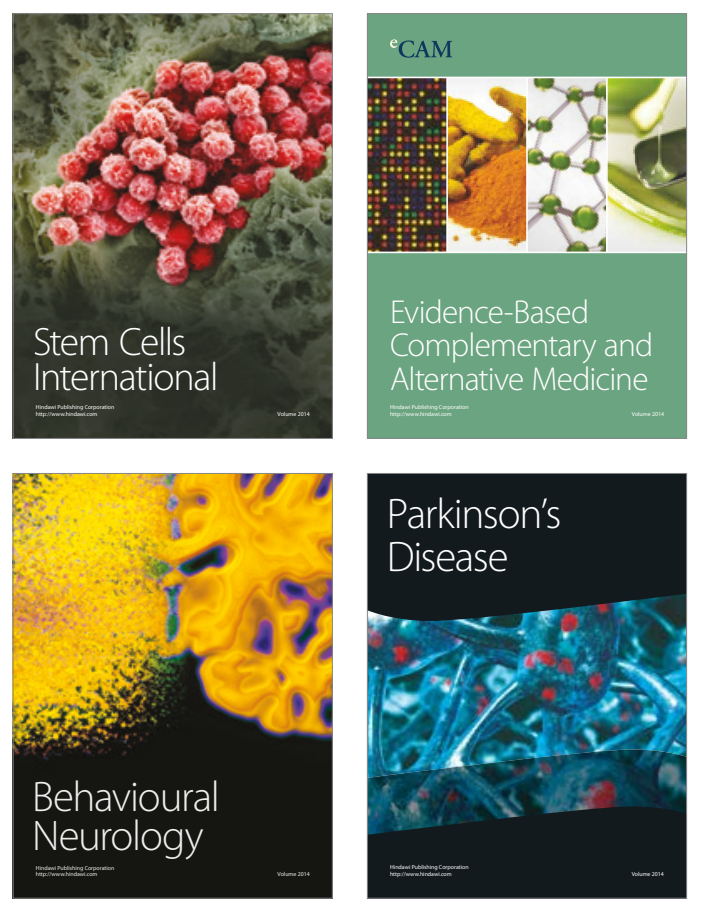
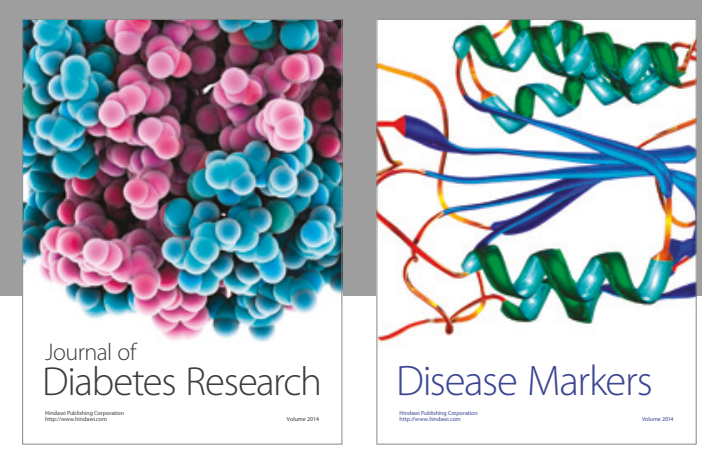

Disease Markers
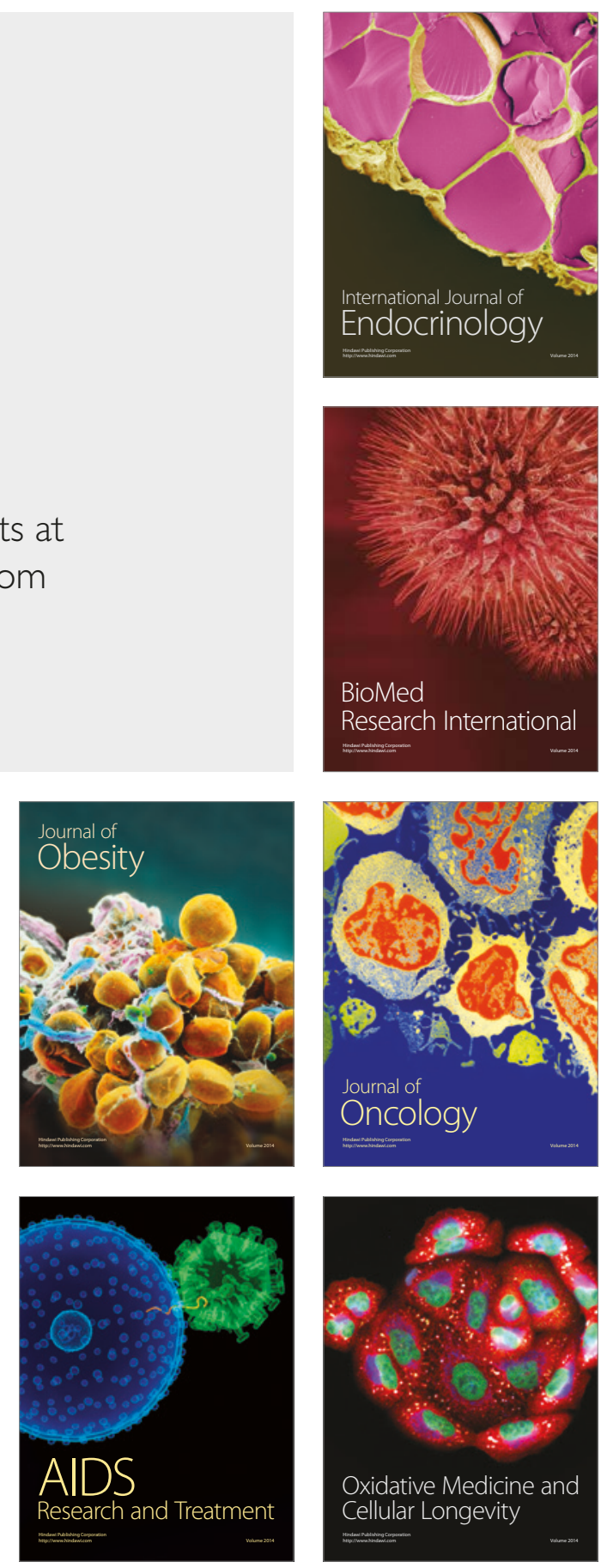